\title{
Methylguanidinium at the Air/Water Interface: A Simulation Study with the Drude Polarizable Force Field
}

\author{
Jian Zhu, Jing Huang* \\ Zhejiang Provincial Laboratory of Life Sciences and Biomedicine, Key \\ Laboratory of Structural Biology of Zhejiang Province, School of Life Sciences, \\ Westlake University, 18 Shilongshan Road, Hangzhou, Zhejiang 310024, China. \\ Institute of Biology, Westlake Institute for Advanced Study, \\ 18 Shilongshan Road, Hangzhou, Zhejiang 310024, China. \\ *huangjing@westlake.edu.cn
}

(Dated: September 18, 2020)

\begin{abstract}
Methylguanidinium is an important molecular ion which also serves as the model compound for arginine side chain. We studied the structure and dynamics of methylguanidium ion at the air/water interface by molecular dynamics simulations employing the Drude polarizable force field. We found out that methylguanidinium accumulate on the interface with a majority adopting tilted conformations. We also demonstrated that methylguanidinium and guanidinium ions have different preference towards the air/water interface. Our results illustrate the importance to explicitly include the electronic polarization effects in modeling interfacial properties.
\end{abstract}




\section{INTRODUCTION}

Guandidinium $\left(\mathrm{Gdm}^{+}\right)$and methylguanidinium $\left(\mathrm{M}-\mathrm{Gdm}^{+}\right)$are important molecular ions with unique physicochemical natures. They assume a planar structure as the three nitrogen lone pairs conjugate with the empty $p$-orbital of the central carbon atom. The lone pairs of the nitrogen atoms are poor hydrogen-bond (H-bond) acceptors while the $\mathrm{N}-\mathrm{H}$ groups oriented in the molecular plane are hydrogen-bond donors, which leads to anisotropic hydration structures. For the $\mathrm{Gdm}^{+}$ion, it is hydrophilic around its plane edge, but hydrophobic in the two perpendicular directions. For the

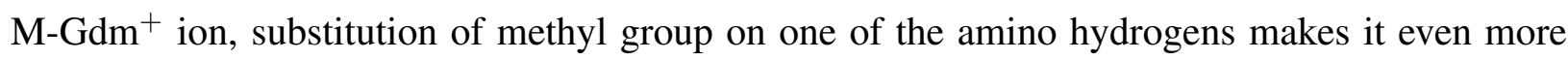
anisotropic. ${ }^{1} \mathrm{Gdm}^{+}$and $\mathrm{M}-\mathrm{Gdm}^{+}$are often studied as the model compounds for arginine side chain, and such an anisotropic electronic structure has implication in its interaction with other amino acids ${ }^{2-4}$ and small molecules. ${ }^{5}$ The particular importance of arginine in liquid-liquid phase separation has also been recently recognized. ${ }^{6}$

Specific ion effects on aqueous interfaces have long been of interests. ${ }^{7,8}$ Early studies date back to Onsager and Samaras, who elucidated the mechanism of ion depletion from the interfacial region using the Debye \& Hückel and the dielectric continuum theory (DH-DCT). ${ }^{9}$ In the 1990s Perera and Berkowitz found that heavy halide ions such as $\mathrm{Br}^{-}$and $\mathrm{I}^{-}$would accumulate at the air/water interface employing molecule dynamics (MD) simulations with a polarizable force field (FF). ${ }^{10,11}$ Since then, the propensity of simple ions at the interface has been studied by a variety of surface-sensitive experimental ${ }^{12-15}$ and theoretical approaches. ${ }^{16-21}$ The specific effects that ions can play on protein stabilities ${ }^{22}$ and chemical reactions at the interface ${ }^{23}$ have also been realized.

While most of these interfacial studies focus on single ions, the effect of molecular ions on the interfaces can be more complicated. For instance, a major favoring factor of ions' surface preference is the cavitation free energy that roots in their disruption on water structure and hydrogen bonding interactions, and molecular ions can have strongly anisotropic hydration structures. For planar molecular ions such as $\mathrm{Gdm}^{+}$and $\mathrm{M}-\mathrm{Gdm}^{+}$, whether they adopt certain orientational preference at the interface is also interesting. Recently Strazdaite and co-workers used heterodyne-detected vibrational sum frequency generation (HD-VSFG) to examine the methyl stretch vibration of M$\mathrm{Gdm}^{+}$, and found that a large fraction of ions adopt certain tilt angles at the air/water interface ${ }^{24}$. This contrasts with previous simulation studies, ${ }^{25-27}$ which found that $\mathrm{Gdm}^{+}$has a orientational 
preference of being parallel to the air/water interface. Ou et al carried out MD simulations of guandidinium and methylguanidinium chloride solutions using a polarizable CHARMM fluctuating charge force field, ${ }^{28,29}$ and reported that both $\mathrm{Gdm}^{+}$and $\mathrm{M}-\mathrm{Gdm}^{+}$ions deplete from air/water interface and lie parallel to the surface. ${ }^{27}$ Wernersson et al found out guanidinium ions tended to be enriched at the water/air surface if they are oriented parallel to the surface using MD simulations with a refined additive force field. ${ }^{25}$ However, they showed that, for a majority of orientation, $\mathrm{Gdm}^{+}$is depleted in the interface so the orientationally averaged density profile shows no net surface excess. Results from experimental measurements for the surface excess of $\mathrm{Gdm}^{+}$and ${\mathrm{M}-\mathrm{Gdm}^{+}}$are also mixed, with both interface depletion ${ }^{30}$ and accumulation ${ }^{31}$ were reported.

Polarizable models are crucial for molecular simulations to study ion effects at interfaces, as they allow ions to adjust their charge distribution to minimize the electrostatic self energy cost associated with moving into interfacial regions that have different permittivities on the two sides. Levin et al showed that the work to bring a polarizable ion from bulk water to the air/water surface is one order of magnitude smaller than a nonpolarizable ion. ${ }^{32,33}$ There exist a variety of methods to explicitly model the electronic polarization effects in force fields, including the point induced dipole model, the classical Drude oscillator model, and the fluctuating charge model. During the past decade, substantial progress has been achieved in systematic parametrization of biomacromolecules for polarizable FFs, most notably for the AMOEBA force field that employes the permenant multipole and induced dipole model ${ }^{34,35}$ and the Drude FF that employs the Drude oscillator model. ${ }^{36,37}$ In a recent work, the equivalency of the point induced dipole model and the Drude model is demonstrated by mapping the Drude FF into an AMOEBA-like multipole and induced dipole model. ${ }^{38}$

In the present work, we carry out extensive MD simulations with the Drude polarizable force field to study the structure and dynamics of $\mathrm{M}-\mathrm{Gdm}^{+}$ions at the air/water interface. In the Drude FF, explicit polarizability is introduced by attaching an auxiliary Drude particle carrying a fixed partial charge to its parent atom with a harmonic spring. The position of the Drude particle is determined by the balance of the forces inserted by the external electric field and the harmonic spring, and thus accounts for induced polarization. ${ }^{39}$ The Drude FF has been extensively parametrized for biomolecules including proteins, ${ }^{40,41}$ nucleic acids, ${ }^{42-44}$ carbohydrates ${ }^{45,46}$ and lipids, ${ }^{47}$ and have demonstrated its advantage in modeling the cooperativity in protein folding ${ }^{48}$ and predicting 
water-octanol partition coefficients. ${ }^{49}$ The parameters of $\mathrm{M}-\mathrm{Gdm}^{+}$used in this work are identical to those of Arg in the Drude protein FF. ${ }^{41}$ Our simulation results correlate well with various experimental measurements and provide insights into specific interfacial effects of molecular ions.

The manuscript is organized as follows. Details of MD simulations and trajectory analysis will be provided in the Methods section. In the Results section, the density profiles of solutes and solvent are first examined, followed by analysis of orientational preference of $\mathrm{M}-\mathrm{Gdm}^{+}$ions at the interface. Molecular structures, induced dipole moments, and simulations with different counteranions will also be presented in this section. The manuscript ends with a short discussion and conclusion.

\section{METHODS}

\section{A. MD simulations}

All the simulations and analysis were performed using CHARMM ${ }^{50}$ To setup a M-Gdm ${ }^{+}$chloride solution system with $3 \mathrm{~m}$ molar concentration, $391 \mathrm{M}-\mathrm{Gdm}^{+}$and $391 \mathrm{Cl}^{-}$ions were solvated in a $60 \AA \times 60 \AA \times 60 \AA$ cubic water box consisted of 5619 water molecules. 6 ns NPT simulations were carried out at $300 \mathrm{~K}$ and 1 atm with periodic boundary conditions, and the averaged volume of the last $5 \mathrm{~ns}$ was used to determine the box dimension for following $1 \mathrm{~ns}$ NVT equilibrium simulations. After that, rectangular simulation systems were built by keeping two dimensions ( $x$ and $y$ ) same and triple the third $(z)$ dimension, creating two air/water interfaces. 20 ns MD simulations were then carried out in the NVT ensemble as the production run. In addition, $\mathrm{GdmCl}$ systems were simulated to compare $\mathrm{M}-\mathrm{Gdm}^{+}$with $\mathrm{Gdm}^{+}$, and systems of $3 \mathrm{~m} \mathrm{M-Gdm}{ }^{+}$(or $\mathrm{Gdm}^{+}$) bromide and iodide solution were set up to investigate the effects of counteranions. A summary of all simulation systems and the simulation protocol is provided in the Supporting Information (Table S1 and Fig. S1).

The Drude polarizable FF was used to model all molecules in the simulation systems. In particular, FF parameters for ions were based on Ref. 51 and Ref. 52 while the SWM4-NDP model ${ }^{53}$ was used for water. Electrostatic interactions were calculated by particle mesh Ewald (PME) summation ${ }^{54}$ with screening parameter $\kappa=0.34$. Lennard-Jones interactions were computed with 
a $12 \AA$ cutoff and a switching function from 10 to $12 \AA$. The nonbonded interaction lists were generated using a distance cutoff of $16 \AA$ and updated heuristically. SHAKE algorithm was used to constrain covalent bonds involving hydrogens.

Nose-Hoover thermostats ${ }^{55}$ and modified Anderson-Hoover barostats ${ }^{56}$ were used for the MD simulations, with characteristic response time both set to be $0.1 \mathrm{ps}$. A velocity-Verlet integrator based on the operator-splitting technique was used for MD propagation. ${ }^{57}$ The relative motion between Drude particles and their parent atoms was further coupled to a thermostat with temperature of $1 \mathrm{~K}$ and characteristic response time of $0.005 \mathrm{ps}$. MD timestep was set to be $1 \mathrm{fs}$, and coordinates were saved every $1 \mathrm{ps}$.

As a comparison, non-polarizable simulations were also carried out using the CHARMM additive FF for ions and the CHARMM TIP3P model for water. ${ }^{58}$ All simulation parameters were the same with polarizable simulations except $2 \mathrm{fs}$ timestep was used.

\section{B. Analysis of MD trajectories}

Interfacial area. To compute the number density ( $\rho$ ) for each component, we discretized the simulation system by z coordinates creating $0.5 \AA$ slices, and counted the number of corresponding molecules per unit volume in each slab by considering their centers of mass. Center of mass of the full simulation system is set to be the origin of the coordinate system. Relative number density $\left(\rho / \rho_{\text {bulk }}\right)$ was then computed by normalization with the bulk density value that was calculated using the -1 to $1 \AA$ slices at the box center, and averaging over the two equivalent halves of the slab system. Relative chemical excess density was computed as the relative number density of each component divided by that of water for each $0.5 \AA$ slice.

Gibbs dividing surface (GDS), defined as the interfacial plane where the surface excess of solvent is zero, was computed as the xy-plane where the relative number density of water equals 0.5 . The width of the interface region was then computed as twice the distance between GDS and outermost boundary of interface. We defined the outermost boundary as the xy-plane where the relative density of water is less than 0.01 in this study. 
Molecular orientation. The key result from HD-VSFG measurements is an ensemble averaged $D$ value determined by the orientation of the $\mathrm{M}-\mathrm{Gdm}^{+}$methyl group at the air/water interface ${ }^{24}$

$$
D=\frac{\left\langle\cos ^{3} \theta_{\mathrm{CH}_{3}}\right\rangle}{\left\langle\cos \theta_{\mathrm{CH}_{3}}\right\rangle}
$$

, where $\theta_{\mathrm{CH}_{3}}$ is the angle between the methyl $\mathrm{C}_{3}$ axis and the surface normal. Another variable to describe the molecular orientation of $\mathrm{M}-\mathrm{Gdm}^{+}$at the interface would be the angle $\theta_{\text {MP }}$ between the molecular plane and the surface normal. We note that $\theta_{\mathrm{CH}_{3}}$ as the angle between two vectors ranges from 0 to $180^{\circ}$, while $\theta_{\mathrm{MP}}$ as the angle between a plane and a vector, ranges from 0 to $90^{\circ}$.

Surface tension. The surface tension of every system was calculated from their MD trajectories as

$$
\gamma=\frac{L_{z}}{2}\left(P_{z z}-\frac{P_{x x}+P_{y y}}{2}\right)
$$

, where $P_{x x}, P_{y y}$, and $P_{z z}$ are the diagonal elements of the internal pressure tensor, and $L_{z}$ is the length of the simulation cell in the direction perpendicular to the surface ${ }^{59}$. Division by 2 accounted for the fact that there were two interfaces in each simulation system.

\section{RESULTS}

\section{A. Density Profiles}

Number density profiles of ions and water along the z-dimension computed from $20 \mathrm{~ns}$ MD simulations of $3 \mathrm{~m} \mathrm{M}-\mathrm{GdmCl}$ solution with the Drude polarizable and CHARMM additive FFs are presented in Fig. 1. The air/water interface of the Drude simulation system centers at $z=29.4$ $\AA$ as indicated by the position of GDS and spans along $\mathrm{z}$ axis for a width of $9.2 \AA$. For the additive simulation system, GDS locates at $z=30.6 \AA$ and the width of interface equals $7.9 \AA$. As a comparison, the widths of air/water interfaces in bulk water systems are $12.9 \AA$ and $9.3 \AA$ with the Drude SWM4-NDP and the additive TIP3P water model, respectively (Fig. S2). The relative number density of $\mathrm{M}-\mathrm{Gdm}^{+}$shows an increase at and just below the interface with the Drude FF. The maximal number density of $\mathrm{M}-\mathrm{Gdm}^{+}$is 1.16 times of the bulk value located at $2.4 \AA$ below GDS, and equals 0.89 of the bulk density at the Gibbs dividing surface. With the CHARMM additive FF, the density of $\mathrm{M}-\mathrm{Gdm}^{+}$decreases monotonously to the outermost boundary after a 
small rise at about $9 \AA$ below GDS. For chlorine anion, there is a significant change of ion density from bulk to the interface with the Drude FF. Interfacial accumulation with relative number density as large as 1.4 is observed in the air/water interface, complemented by a slight depletion in the subinterfacial region. In contrast, significant interfacial depletion of $\mathrm{Cl}^{-}$ions is observed in the additive simulation.

Drude

a)

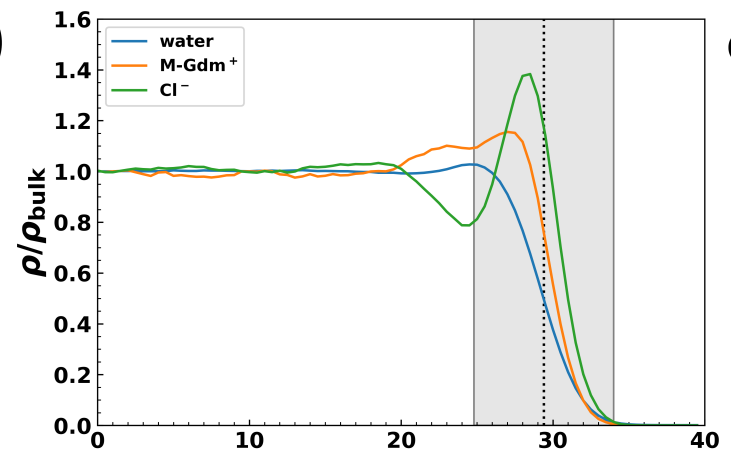

b)

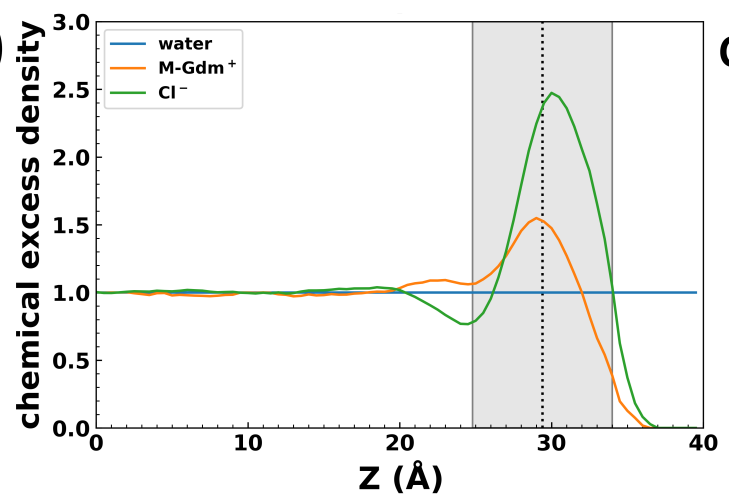

additive
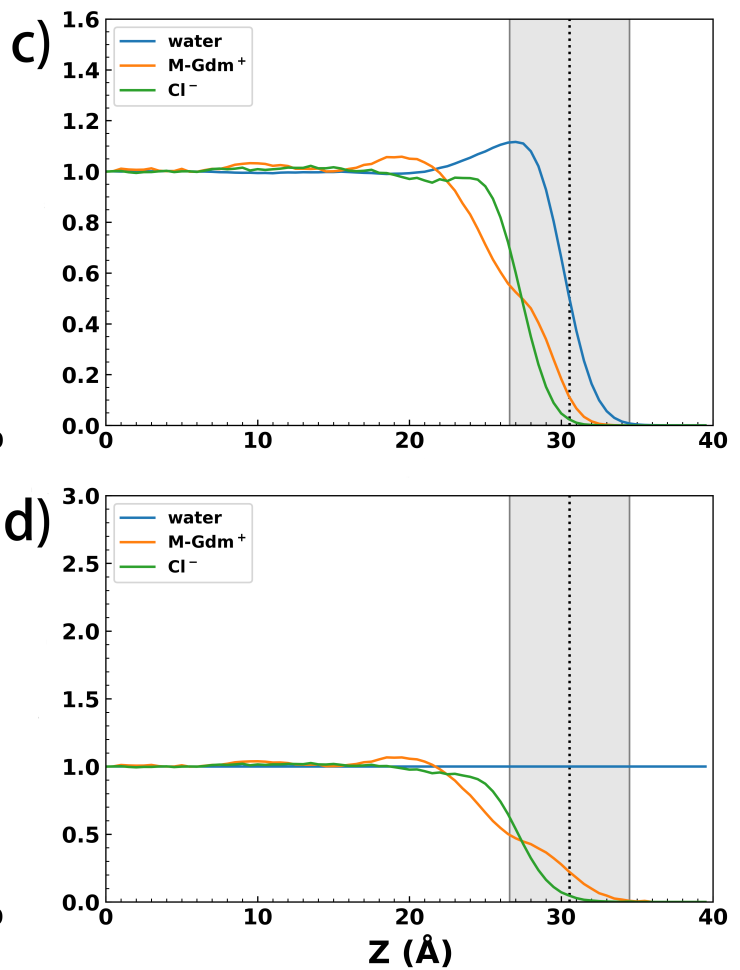

FIG. 1. Relative number density profiles (a,c) and relative chemical excess density profiles (b,d) of water (blue), $\mathrm{M}-\mathrm{Gdm}^{+}$(red) and $\mathrm{Cl}^{-}$(green) in $3 \mathrm{~m} \mathrm{M}-\mathrm{GdmCl}$ solution simulated with Drude polarizable and CHARMM additive FFs. GDS is indicated by dashed lines, and the interface is depicted by the grey area.

Chemical excess density, defined as the ratio of the number of solutes to that of water within a local region, is important to understand interfacial chemical reactions and used here to present the interfical accumulation or depletion of ions. The relative chemical excess density, $\frac{\rho^{\text {ion }} / \rho_{\text {bulk }}^{\text {ion }}}{\rho^{\text {water }} / \rho_{\text {bulk }}^{\text {watk }}}$, are calculated from the Drude and additive simulations and plotted in Fig. $1 \mathrm{~b}$ and $1 \mathrm{~d}$. For $3 \mathrm{~m}$ $\mathrm{M}-\mathrm{GdmCl}$ solution with the Drude polarizable force field, the chemical excess densities of M$\mathrm{Gdm}^{+}$and $\mathrm{Cl}^{-}$can be 1.6 and 2.5 times of bulk values, and their peaks are both located at the 
vicinity of GDS. Integrating over the whole interfacial region, the relative chemical excess densities of $\mathrm{M}-\mathrm{Gdms}^{+}$and $\mathrm{Cl}^{-}$ions equal 1.31 and 1.47 respectively, indicating strong accumulation of $\mathrm{M}-\mathrm{Gdm}^{+}$and $\mathrm{Cl}^{-}$ions at the air/water interface. On the contrary, in the additive simulation the relative chemical excess densities of $\mathrm{M}-\mathrm{Gdms}^{+}$and $\mathrm{Cl}^{-}$ions are 0.10 and 0.02 at GDS respectively, and 0.39 and 0.30 on the average over the whole interfacial region, indicating strong interfacial depletion of both cations and anions and consistent with the previous MD studies of ${\mathrm{M}-\mathrm{Gdm}^{+}}^{+}\left(\right.$or $\left.\mathrm{Gdm}^{+}\right)$chloride solution with non-polarizable force field. ${ }^{25-27}$

\section{B. Orientational Preference at the Interface}

While simple ions assume spherical shape, $\mathrm{M}-\mathrm{Gdm}^{+}$is a molecular ion that has a planar structrue. The orientation of its molecular plane at the interface thus becomes an interesting topic, and has been investigated by many theoretical and experimental studies. A recent VSFG experiment found that a large fraction of $\mathrm{M}-\mathrm{Gdm}^{+}$ions have an angle relative to the interface, ${ }^{24}$ conflicting with previous MD simulaitons which predicted that most interficial $\mathrm{M}-\mathrm{Gdm}^{+}$ions lie parellel to the interface. ${ }^{25-27}$ Key observable from the VSFG measurement is the ensemble averaged $D$ value determined by the orientation of the $\mathrm{M}-\mathrm{Gdm}^{+}$methyl group with respect to the air/water interface. Specifically, $D_{\text {exp }}$ equals $0.5 \pm 0.06$. By assuming a partial-Gaussian distribution of molecular tilt angle Ref. 24 concluded that at least $50 \%$ of interfical $\mathrm{M}-\mathrm{Gdm}^{+}$orients at an angle $>20^{\circ}$ with respect to the surface plane. From our MD simulations, $D_{\text {calc }}$ values from Eq. (1) equal $0.45 \pm 0.01$ with the Drude FF and $0.48 \pm 0.01$ with the CHARMM additive FF, respectively. Both agree with the experimental value within the uncertainties.

Although both the Drude and the additive simulations lead to similar ensemble averaged $D$ values, the distributions of $\theta_{\mathrm{CH}_{3}}$ are actually quite different as evident in Fig. 2. We compute the orientationally resolved number density profile $g\left(z, \theta_{\mathrm{CH}_{3}}\right)$ defined as

$$
g(z, \theta)=\int_{z}^{z+\Delta z} \int_{\theta}^{\theta+\Delta \theta} d\left(z^{\prime}, \theta^{\prime}\right) \mathrm{d} \theta^{\prime} \mathrm{d} z^{\prime}
$$

, where $\Delta \theta$ and $\Delta z$ are set to $1^{\circ}$ and $1.0 \AA$, respectively, and $d\left(z^{\prime}, \theta^{\prime}\right)$ is the local number density function defined as

$$
d\left(z^{\prime}, \theta^{\prime}\right)=\lim _{\substack{\Delta z^{\prime} \rightarrow 0 \\ \Delta \theta^{\prime} \rightarrow 0}} \frac{n\left(z^{\prime}, \theta^{\prime}, \Delta z^{\prime}, \Delta \theta^{\prime}\right)}{s \cdot \Delta \theta^{\prime} \cdot \Delta z^{\prime}}
$$


Drude

a)
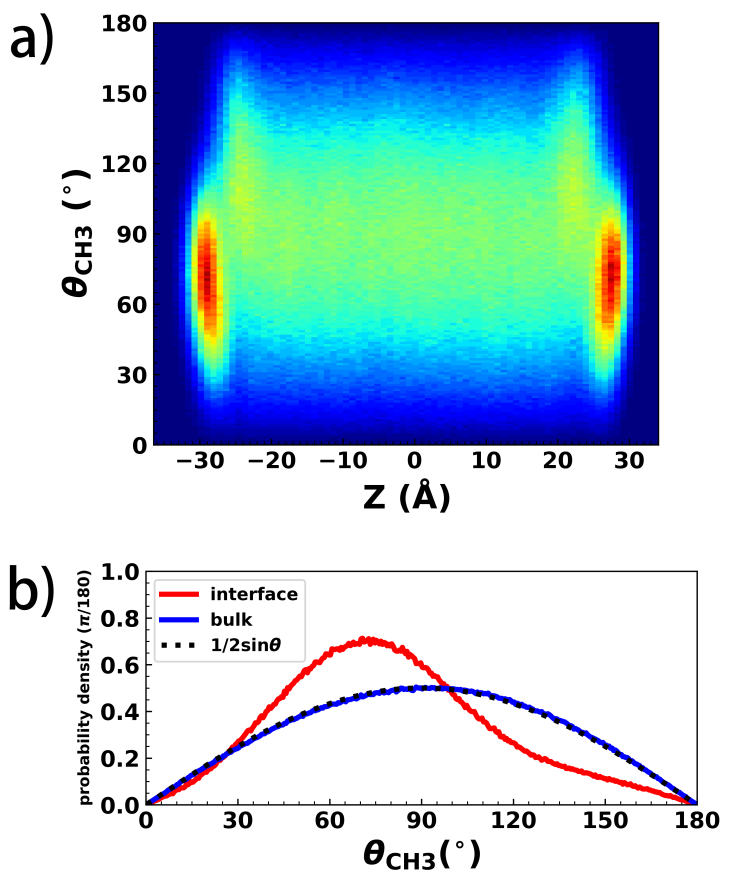

additive
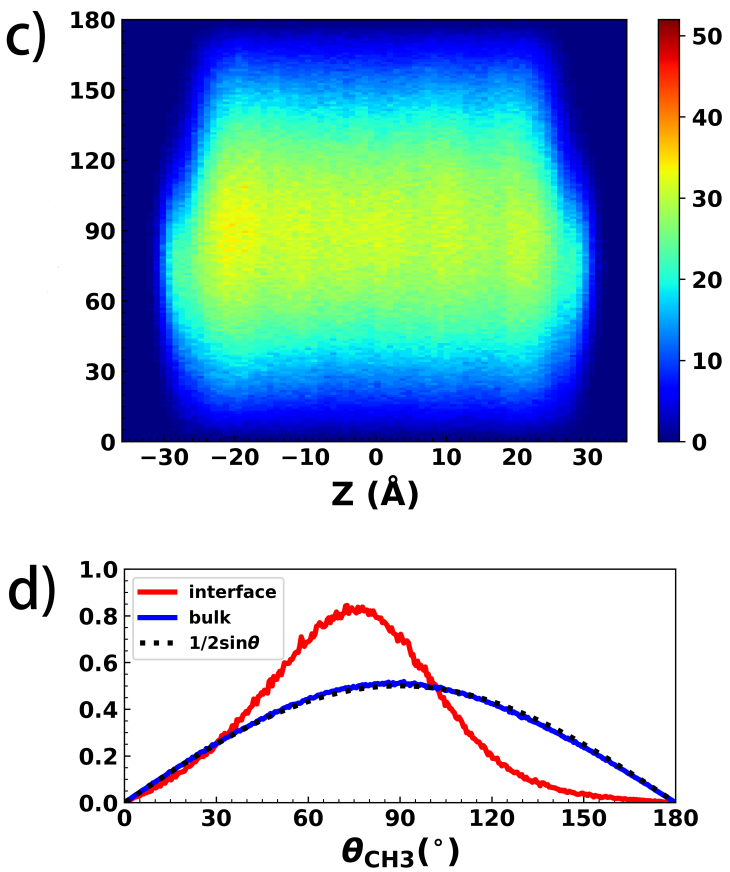

FIG. 2. The orientationally resolved number density profile $g\left(z, \theta_{\mathrm{CH}_{3}}\right)$ of $\mathrm{M}-\mathrm{Gdm}^{+}$ions (top panels, a and c) as a function of its orientation $\theta_{\mathrm{CH}_{3}}$ and its distance from box center $(z=0 \AA)$, together with the probability density profile $\rho\left(\theta_{\mathrm{CH} 3}\right)$ of $\mathrm{M}-\mathrm{Gdm}^{+}$ions at the interface and in the bulk, as well as a standard function of $1 / 2 \sin \theta$ (bottom panels, $\mathrm{b}$ and $\mathrm{d}$ ) as a function of its orientation $\theta_{\mathrm{CH} 3}$ in $3 \mathrm{~m} \mathrm{M}-\mathrm{GdmCl}$ solution with the Drude polarizable (left panels, a and b) and the CHARMM additive (right panels, $\mathrm{c}$ and d) FFs.

, where $n\left(z^{\prime}, \theta^{\prime}, \Delta z^{\prime}, \Delta \theta^{\prime}\right)$ is the number of ions whose orientational angle $\theta_{\mathrm{CH}_{3}}$ ranges from $\theta^{\prime}$ to $\theta^{\prime}+\Delta \theta^{\prime}$ and $z$ coordinate ranges from $z^{\prime}$ to $z^{\prime}+\Delta z^{\prime}$, and $s$ is the area of xy plane of the simulation box. The $2 \mathrm{D}$ distribution $g\left(z, \theta_{\mathrm{CH}_{3}}\right)$ of $3 \mathrm{~m} \mathrm{M}-\mathrm{Gdm}^{+}$chloride solution calculated from the Drude and the additive simulations are plotted in Fig. $2 \mathrm{a}$ and $2 \mathrm{c}$, respectively. In the Drude simulation, the orientationally resolved number density profile in the bulk region (from $-24.8 \AA$ to $24.8 \AA$ ) is symmetric along $\theta_{\mathrm{CH}_{3}}=90^{\circ}$, indicating no orientational preference in the bulk. At interface, there is a significant enrichment of $\theta_{\mathrm{CH}_{3}}$ in the region from $30^{\circ}$ to $100^{\circ}$ with peak at $70^{\circ}$. The orientation distribution in bulk and at interface is further analyzed using 1D probabilty density $\rho\left(\theta_{\mathrm{CH}_{3}}\right)$. As shown in Fig. $2 b$, the probabilty density function $\rho\left(\theta_{\mathrm{CH}_{3}}\right)$ in the bulk is almost identical to $1 / 2 \sin \theta_{\mathrm{CH}_{3}}$, which means that the orientational distribution of $\mathrm{M}-\mathrm{Gdm}^{+}$methyl $\mathrm{C}_{3}$ axis at each azimuthal angle is an uniform distribution. In contrast, at the air/water interface the most 
probable $\theta_{\mathrm{CH}_{3}}$ angle is $72^{\circ}$, and $65.6 \%$ of $\mathrm{M}-\mathrm{Gdm}^{+}$ions have an angle $\theta_{\mathrm{CH}_{3}}<90^{\circ}$ (methyl group pointing towards the vacuum side). In the simulation using additive FF, M-Gdm ${ }^{+}$ions are strongly depleted at the air/water interface for all $\theta_{\mathrm{CH}_{3}}$ angles (Fig. 2c). Nevertheless, similar difference of $\rho\left(\theta_{\mathrm{CH}_{3}}\right)$ distribution between the bulk and the interface environment is observed (Fig. $2 \mathrm{~d}$ ). $\theta_{\mathrm{CH}_{3}}$ in the bulk follows an uniform distribution, while $\rho\left(\theta_{\mathrm{CH}_{3}}\right)$ at the interface has a peak at $75^{\circ}$ and the probability of $\theta_{\mathrm{CH}_{3}}<90^{\circ}$ equals $71.3 \%$.

a)

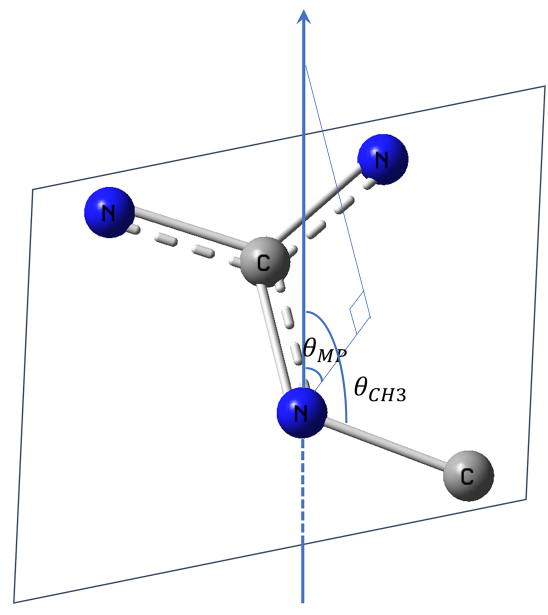

b)

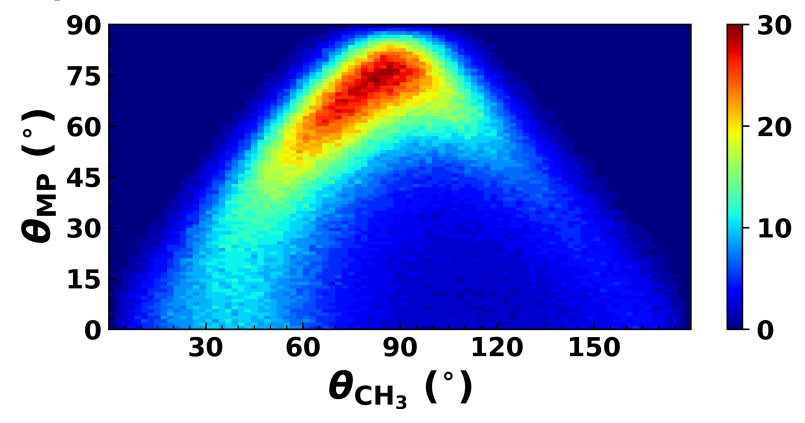

FIG. 3. a: Comparison between two representation of molecular orientation: $\theta_{\mathrm{MP}}$ and $\theta_{\mathrm{CH}_{3}}$. b: The number count profile $g\left(\theta_{\mathrm{MP}}, \theta_{\mathrm{CH}_{3}}\right)$ of interficial M-Gdm ${ }^{+}$ions as a function of $\theta_{\mathrm{CH}_{3}}$ and $\theta_{\mathrm{MP}}$ in $3 \mathrm{~m} \mathrm{M-GdmCl}$ solution modeled with the Drude polarizable FF.

While $\theta_{\mathrm{CH}_{3}}$ directly connects with experimental spectroscopic measurement, another variable to describe the orientation of $\mathrm{M}-\mathrm{Gdm}^{+}$would be the angle $\theta_{\mathrm{MP}}$ between the molecular plane and the normal of the interface plane. $\theta_{\mathrm{MP}}$ ranges from 0 to $90^{\circ}$ and is mathematically different from $\theta_{\mathrm{CH}_{3}}$ (see Fig. 3a). $\theta_{\mathrm{MP}}$ is an angle between a vector and a plane, which is different from the angle $\theta_{\mathrm{CH} 3}$ between this vector and a certatin vector in the plane. Given a fixed $\theta_{\mathrm{MP}}$, when the molecular plane of $\mathrm{M}-\mathrm{Gdm}^{+}$rotates along its normal, $\theta_{\mathrm{CH}_{3}}$ could vary from $\theta_{\mathrm{MP}}$ to $180^{\circ}-\theta_{\mathrm{MP}}$. We calculate the number count profile $g\left(\theta_{\mathrm{MP}}, \theta_{\mathrm{CH}_{3}}\right)$ for $\mathrm{M}-\mathrm{Gdm}^{+}$ions at the air/water interface from the Drude simuilation and present it in Fig. 3b. For a given $\theta_{\mathrm{MP}}$, the relationship $\theta_{\mathrm{MP}} \leq \theta_{\mathrm{CH}_{3}} \leq 180^{\circ}-\theta_{\mathrm{MP}}$ is strictly satisfied. Furthermore, a large fraction of $\mathrm{M}-\mathrm{Gdm}^{+}$ions, especially those with smaller tilt angles, have their $\theta_{\mathrm{CH}_{3}}$ among $\theta_{\mathrm{MP}} \pm 5^{\circ}$, indicating limited out-of-plane rocking motion of 
the methyl group. Our results suggest that it is reasonable to use $\theta_{\mathrm{CH}_{3}}$ to represent the molecular orientation at interface.

We also compute and analyze the orientationally resolved number density profile using $\theta_{\mathrm{MP}}$ instead of $\theta_{\mathrm{CH}_{3}}$. As illustrated in Fig. S3, the 1D probability density profiles $\rho\left(\theta_{\mathrm{MP}}\right)$ in bulk follow the uniform distribution of orientations at each azimuthal angle, i.e. $\rho\left(\theta_{M P}\right)=\cos \theta_{M P}$. At interface, $\rho\left(\theta_{\mathrm{MP}}\right)$ has the peak value at about $70^{\circ}$ and mean at about $44.3^{\circ}$ in the Drude simulation. If we consider a $\mathrm{M}-\mathrm{Gdm}^{+}$ion lying parallel to the interface when its $\theta_{\mathrm{MP}}$ is among $80^{\circ}$ to $90^{\circ}$, $5.2 \%$ of $\mathrm{M}-\mathrm{Gdm}^{+}$ions are parallel on the air/water interface. Alternatively if parallel $\mathrm{M}-\mathrm{Gdm}^{+}$is defined as $\theta_{\mathrm{MP}}$ ranges from $70^{\circ}$ to $90^{\circ}$, then $18.4 \%$ of $\mathrm{M}-\mathrm{Gdm}^{+}$ions are parallel.

One of the shortcoming of using $\theta_{\mathrm{MP}}$ is that it is a certain azimuthal angle, rather than a $\theta_{\mathrm{MP}}$, that corresponds to the orientation of a M-Gdm ${ }^{+}$ion in a one-to-one way, i.e. for $\mathrm{M}-\mathrm{Gdm}^{+}$ions with a given $\theta_{\mathrm{MP}}$, there are many corresponding azimuthal angles, of which the measure (density of

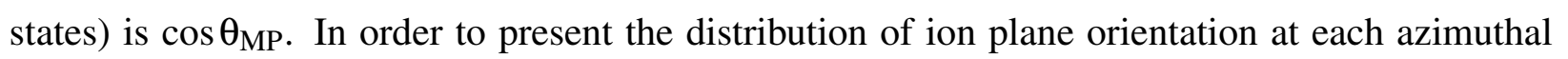
angle, another number density profile $g^{\prime}\left(z, \theta_{\mathrm{MP}}\right)$ is defined as

$$
g^{\prime}(z, \theta)=\int_{z}^{z+\Delta z} \int_{\sin \theta}^{\sin \theta+\Delta \sin \theta} d^{\prime}\left(z^{\prime}, \theta^{\prime}\right) \mathrm{d} \sin \theta^{\prime} \mathrm{d} z^{\prime}
$$

, where $\Delta \sin \theta$ and $\Delta z$ are set to $1 / 180$ and $1.0 \AA$, respectively, and $d^{\prime}\left(z^{\prime}, \theta^{\prime}\right)$ is defined as

$$
d^{\prime}\left(z^{\prime}, \theta^{\prime}\right)=\lim _{\substack{\Delta z^{\prime} \rightarrow 0 \\ \Delta \sin \theta^{\prime} \rightarrow 0}} \frac{n\left(z^{\prime}, \theta^{\prime}, \Delta z^{\prime}, \Delta \sin \theta^{\prime}\right)}{s \cdot \Delta \sin \theta^{\prime} \cdot \Delta z^{\prime}}
$$

, where $n\left(z^{\prime}, \theta^{\prime}, \Delta z^{\prime}, \Delta \sin \theta^{\prime}\right)$ is the number of ions whose orientational angle $\theta_{\mathrm{MP}}$ ranges from $\theta^{\prime}$ to $\arcsin \left(\sin \theta^{\prime}+\Delta \sin \theta^{\prime}\right)$ and $z$ coordinate ranges from $z^{\prime}$ to $z^{\prime}+\Delta z^{\prime} . d^{\prime}(z, \theta)=d(z, \theta) / \cos \theta$. If $\Delta \sin \theta \rightarrow 0$ and $\Delta \theta \rightarrow 0$, then $g(z, \theta) \rightarrow d(z, \theta)$ in Eq. (3) and $g^{\prime}(z, \theta) \rightarrow d^{\prime}(z, \theta)$ in Eq. (5), respectively. Hence $g^{\prime}(z, \theta)$ is a locally resolved number density function of ions with the $\mathrm{z}$ component of coordinates at $z$ and the orientation at any azimuthal angle that satisfies $\theta_{\mathrm{MP}}=\theta$. The major difference between $g$ and $g^{\prime}$ can be understood as different variables $(\theta$ and $\sin \theta)$ are used in the $y$-axis such that there is stretching in low $\theta$ region and compression in large $\theta$ region in $g^{\prime}$ compared to $g$ (Fig. S3b), which is also visible as the difference between $\rho\left(\theta_{\text {MP }}\right)$ and $\rho^{\prime}\left(\theta_{\mathrm{MP}}\right)=\rho\left(\theta_{\mathrm{MP}}\right) / \cos \left(\theta_{\mathrm{MP}}\right)$ (Fig. S3c). The physical variable related to $g^{\prime}(z, \theta)$ is the potential of mean force $(\mathrm{PMF}) w(z, \theta)$ for a constrained ion with a given azimuthal angle satisfied $\theta_{\mathrm{MP}}=\theta$ 
through the relation

$$
w(z, \theta)=-k_{\mathrm{B}} T \ln g^{\prime}(z, \theta)+\mathrm{cons}
$$

, similarly the 1D PMF $w(\theta)$ for a constrained ion with a given azimuthal angle at the interface could be computed as

$$
w(\theta)=-k_{\mathrm{B}} T \ln \rho^{\prime}(\theta)+\mathrm{cons}
$$

. Both equations are applicable to infinitely diluted solutions, and approximately satisfied for 3 m solutions. Based on $\rho^{\prime}\left(\theta_{\mathrm{MP}}\right)$ and $w\left(\theta_{\mathrm{MP}}\right)$, the parrallel orientation with respect to the interface $\left(\theta_{\mathrm{MP}}=90^{\circ}\right)$ has lowest potential of mean force among all azimuthal angles for interfacial M$\mathrm{Gdm}^{+}$(Fig. S3d), consistent with previous MD simulation results. ${ }^{27}$ However, taking the measure being $\cos \theta_{\mathrm{MP}}$ into consideration $\left(\cos \left(\theta_{\mathrm{MP}}=90^{\circ}\right)=0\right)$, the probabilty density of $\mathrm{M}-\mathrm{Gdm}^{+}$ions with $\theta_{\mathrm{MP}}=90^{\circ}$ is nearly zero. Direct counting the number of ions leads to the observation that

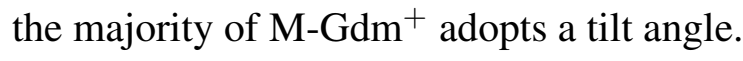

\section{Difference in Interfacial Preference between M-Gdm ${ }^{+}$and Gdm ${ }^{+}$}

MD simulations with the Drude polarizable FF suggest that $\mathrm{M}-\mathrm{Gdm}^{+}$ions accumulate at the air/water interface. A common experimental method to determine the surface excess or depletion of ions is based on surface tension, such that increasing surface tension with ion concentration indicates surface depletion and decreasing surface tension indicates accumulation. We found that, however, such argument for the interfacial preference of $\mathrm{M}-\mathrm{Gdm}^{+}$is often based on the surface tension measurement of guanidinium. ${ }^{30}$ For $\mathrm{Gdm}^{+}$, surface tension of the air/water interface increases with concentration, which suggests the ions are depleted from the interface. To compare with the experimental data, we set up interfacial systems for $\mathrm{Gdm}^{+}$chloride solution with a variety of concentrations, and performed 20 ns MD simulations with the Drude FF at each concentration to reveal the relationship between the surface tension and concentrations.

As shown in Fig. 4a, the computed surface tension of the air/water interface increase with the concentration of $\mathrm{Gdm}^{+} \mathrm{Cl}$. Linear regression of calculational tension leads to a slope of 0.57 $\mathrm{dyn} /(\mathrm{cm} \cdot \mathrm{m})$, close to the linear fitting value of 0.53 based on the experimental data. ${ }^{6}$ We note that there is an offset in the absolute value of surface tension between simulation and experiment. Calculation results are consistently about $9.5 \mathrm{dyn} / \mathrm{cm}$ smaller than the experimental values at each 

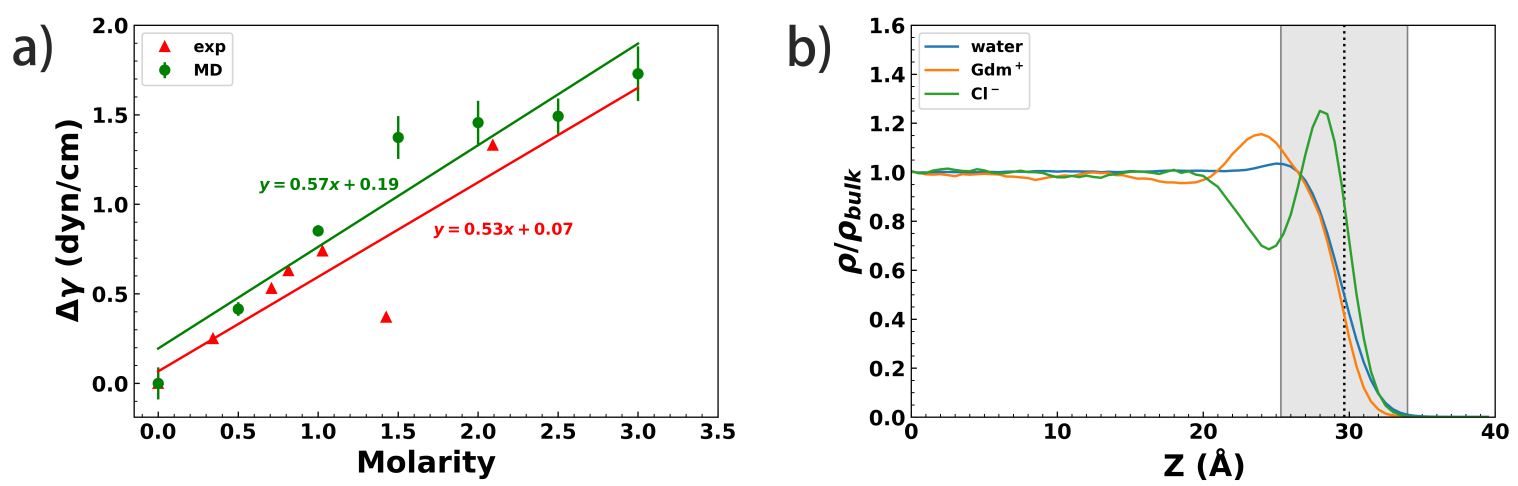

FIG. 4. a) Surface tension change as a function of $\mathrm{Gdm}^{+}$ion concentration, along with linear regression results. b) Normalized number density profiles of water, $\mathrm{Gdm}^{+}$and $\mathrm{Cl}^{-}$in $3 \mathrm{~m} \mathrm{GdmCl}$ solution simulated with the Drude polarizable FF.

concentration, which could be attributed to the SWM4-NDP water model used in the simulation. The air/water surface tension of pure water system computed from Drude simulation at $300 \mathrm{~K}$ is $62.18 \mathrm{dyn} / \mathrm{cm}$, while the experimental value is $71.81 \mathrm{dyn} / \mathrm{cm} .{ }^{60} \mathrm{Such}$ an underestimatation is consistent with previous reported value $(\gamma=67 \pm 4 \mathrm{dyn} / \mathrm{cm}$ at $T=298.15 \mathrm{~K})$ for the SWM4-NDP model. ${ }^{53}$

Our results suggest that the Drude polarizable FF is able to quantitatively reproduce the impact of $\mathrm{Gdm}^{+}$ions on the air/water surface tension. As tension increases with ion concentration, $\mathrm{Gdm}^{+}$ is supposed to be depleted from the surface. Such a depletion is evident from the density profile of $\mathrm{GdmCl}$ solution calculated from the Drude simulation (Fig. $4 \mathrm{~b}$ and Table S2). The relative number density of $\mathrm{Gdm}^{+}$is 0.51 times of the bulk density at GDS, and the chemical excess density over the interfacial region equals 0.97 . We note that the properties of $\mathrm{M}-\mathrm{Gdm}^{+}$are often be inferred from those of $\mathrm{Gdm}^{+}$due to their similarity. However here a counterexample is provided such that $\mathrm{M}-\mathrm{Gdm}^{+}$is accumulated on the air/water interface while $\mathrm{Gdm}^{+}$is depleted. The extra methyl group in $\mathrm{M}-\mathrm{Gdm}^{+}$makes it more hydrophobic than $\mathrm{Gdm}^{+}$, which leads to qualitative difference on the interfacial preference between $\mathrm{M}-\mathrm{Gdm}^{+}$and $\mathrm{Gdm}^{+}$. 


\section{Polarization at the Interface}

Polarization effects are important for the structure and dynamics of ions at interfaces, as ions would adjust their charge distribution to adapt to the inhomogeneous electrostatic environments. We analyze the induced dipole moment (IDM) of ions and water molecules from our simulations with the Drude FF to understand such polarization effects. We note that induced dipole is used because the total dipole moment of a charge molecule (ion) depends on the coordinate system and is thus not well-defined. We also note that in additive simulations, IDMs of all molecules are zero regardless of their environments. Due to the structural asymmetry on two sides of the interface, we consider both the total magnitude $\left(\operatorname{IDM}_{\text {norm }}\right)$ and the signed z component $\left(\operatorname{IDM}_{z}\right)$, and compute their ensemble averages. As depicted in Fig. 5, the magnitudes of induced dipole are almost constant in the bulk, with average $\mathrm{IDM}_{\text {norm }}$ being $1.00 \mathrm{D}$ for $\mathrm{M}-\mathrm{Gdm}^{+}, 2.17 \mathrm{D}$ for $\mathrm{Cl}^{-}$and $0.65 \mathrm{D}$ for water molecules, respectively. It's interesting that for all three molecular species, $\mathrm{IDM}_{\text {norm }}$ begins to change right across into the region of interface that is defined by water density. This demonstrates the sensitivity of molecular polarization on the interfacial environment.

Next we consider the z-component of IDM, and a positive $\mathrm{IDM}_{z}$ means that the induced dipole points towards the vaccum side. In the bulk, average $\mathrm{IDM}_{z}$ equals zero since there is equal probability of postive and negative $\operatorname{IDM}_{z}$. When ions move towards the air/water interface, there is a net effect of induced dipole aligning along the surface normal. At the interface, $\mathrm{IDM}_{z}$ of $\mathrm{M}-\mathrm{Gdm}^{+}$ continuously decreases to $-0.57 \mathrm{D}$, while the $\mathrm{IDM}_{z}$ of $\mathrm{Cl}^{-}$gradually increases to $2.7 \mathrm{D}$. Taking the sign of their charges into consideration, cations and anions both distribute their charge towards the water side of the interface. This result is consistent with previous theoretical consideration that polarizable ions shift their charge towards liquid phase to remain hydrated and reduce the cavitational energy when moving across the interface. ${ }^{32}$

It's also interesting to note the different polarization behavior between simple ion $\left(\mathrm{Cl}^{-}\right)$and molecular ion $\left(\mathrm{M}-\mathrm{Gdm}^{+}\right)$. When a $\mathrm{Cl}^{-}$ion enters the interfacial region, both its total dipole moment and the z-component increase under the stronger, inhomogeneous electric field at interface. With the ion moving closer to the vacuum side, $\mathrm{IDM}_{z}$ increases much faster than $\mathrm{IDM}_{\text {norm }}$ and eventually almost all the dipole moment is distributed in the $\mathrm{z}$ axis to minimize the electrostatic self energy cost (Fig. 5c, d). The average magnitude of IDM of $\mathrm{Cl}^{-}$at GDS equals 2.89 D, while at least 
$85.5 \%(2.47 \mathrm{D})$ is distributed along the surface normal direction. In contrast, the molecular polarizability of a molecular ion is typically anisotropic, such that the direction of its induced dipole is coupled - sometimes strongly - with its geometry. For example the polarizability of M-Gdm ${ }^{+}$has an in-molecular-plane component of $9.09 \AA^{3}$ and an out-of-molecular-plane component of 4.82 $\AA^{3}$, suggesting it is easier to be polarized in directions that are parallel to the molecular plane compared to perpendicular ones. This is illustrated in the decomposition of IDM $_{\text {norm }}$ in Fig. 5a. At GDS, the IDM in the direction perpendicular to $\mathrm{M}-\mathrm{Gdm}^{+}$molecular plane equals $0.27 \mathrm{D}$, while that within the molecular plane is $1.06 \mathrm{D}$. Thus if a $\mathrm{M}-\mathrm{Gdm}^{+}$ion lies parallel on the air/water interface, only a small proportion of its induced dipole moment can be pointed along the surface normal direction. On the other hand, an ion on the interface has a tendency to align its dipole along surface normal. In the Drude simulation, interfacial $\mathrm{M}-\mathrm{Gdm}^{+}$ions orient, and on average about half of their IDM magnitude can be pointed towards the liquid phase (Fig. 5a, b). Thus the anisotropy of molecular polarizability provides additional incentive for $\mathrm{M}-\mathrm{Gdm}^{+}$to adopt a certain tilt angle on the interface.

\section{E. Effects of Anion on Interfacial Properties}

We further investigate how different anions impact the interfacial preference of $\mathrm{M}-\mathrm{Gdm}^{+}$and $\mathrm{Gdm}^{+}$with MD simulations of $3 \mathrm{~m}$ bromide and iodide solutions using the Drude polarizable FF. $\mathrm{Br}^{-}$and $\mathrm{I}^{-}$are more polarizable than $\mathrm{Cl}^{-}$. The atomic polarizabilities of $\mathrm{Cl}^{-}, \mathrm{Br}^{-}$and $\mathrm{I}^{-}$in the Drude FF are 3.969, 5.262, and 7.439 $\AA^{3}$, respectively. Experimentally, it is known that $\mathrm{Br}^{-}$and $\mathrm{I}^{-}$ions have stronger interfacial preference than $\mathrm{Cl}^{-} .{ }^{7}$ As shown in Fig. 6, strong accumulation of $\mathrm{Br}^{-}$and $\mathrm{I}^{-}$towards the air/water interface is observed in the simulations. As a consequence, molecular cations $\left(\mathrm{M}-\mathrm{Gdm}^{+}\right.$and $\left.\mathrm{Gdm}^{+}\right)$are also more likely to be attracted to the interface. There are more $\mathrm{M}-\mathrm{Gdm}^{+}$ions accumulated at the interface, with average relative chemical excess density of 1.96 and 2.25 in the bromide and iodide solutions repectively, compared with that of 1.31 in the chloride solution. Similar interfacial enrichment is observed for $\mathrm{Gdm}^{+}$ions in the bromide and iodide solutions (Fig. 6 b and d), with chemical excess density being 1.71 and 2.11 respectively, compared with that of 0.97 in the chloride solution. Thus $\mathrm{Gdm}^{+}$'s surface preference changes from depletion to accumulation when the corresponding anions change from chloride to bromide and iodide ions. We also note that not only can the interfacial preference of molecular 
$\mathrm{M}-\mathrm{Gdm}^{+}$

a)

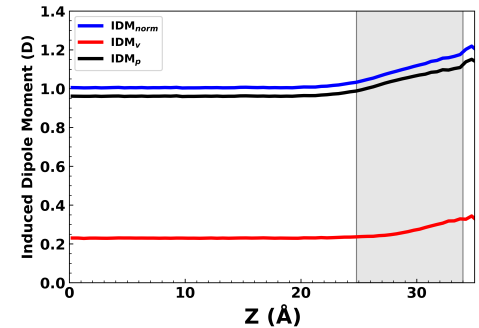

b)

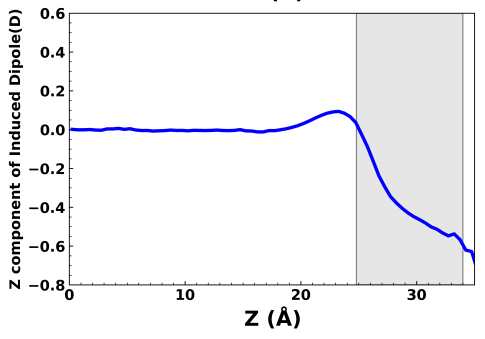

$\mathrm{Cl}^{-}$
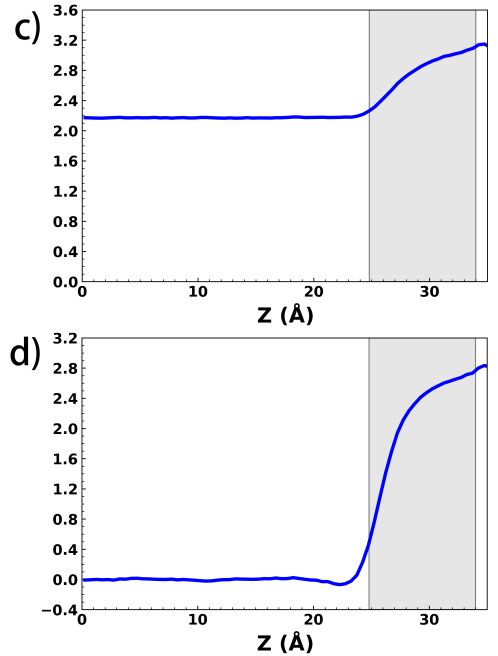
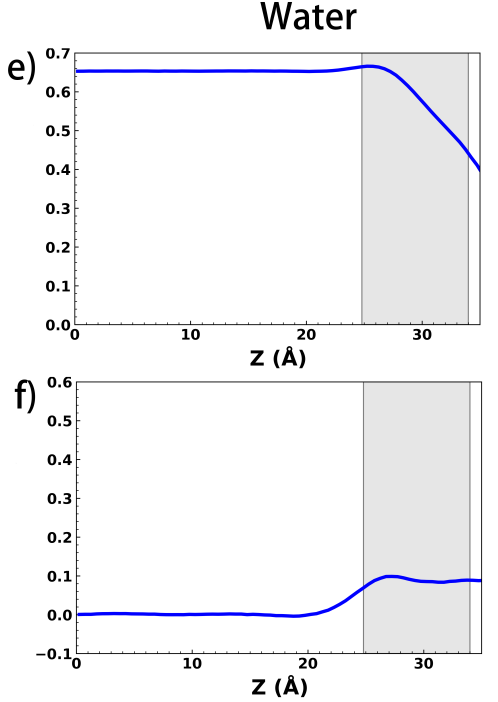

FIG. 5. The average norm (top panels) and signed z component (bottom panels) of induced dipole moment for $\mathrm{M}-G d m^{+}(\mathrm{a}, \mathrm{b}), \mathrm{Cl}^{-}(\mathrm{c}, \mathrm{d})$ ions and water molecules (e, f), as a function of the distance from box center $(z=0 \AA)$ in $3 \mathrm{~m} \mathrm{M}-\mathrm{GdmCl}$ solution simulated with the Drude polarizable force field. Additional decomposition of $\operatorname{IDM}_{n o r m}$ into in-molecular-plane ( $\left.\operatorname{IDM}_{p}\right)$ and out-of-molecular-plane ( $\left.\operatorname{IDM}_{v}\right)$ components for $\mathrm{M}-\mathrm{Gdm}^{+}$is shown in the subfigure a.

cation $\left(\mathrm{M}-\mathrm{Gdm}^{+}\right.$or $\left.\mathrm{Gdm}^{+}\right)$be affected by different counteranions $\left(\mathrm{Cl}^{-}, \mathrm{Br}^{-}, \mathrm{I}^{-}\right)$, but also the interfacial preference of anions is affected by different countercations. Slightly more halide ions $\left(\mathrm{Cl}^{-}, \mathrm{Br}^{-}\right.$or $\left.\mathrm{I}^{-}\right)$accumulate at the air/water interface in the case of $\mathrm{M}-\mathrm{Gdm}^{+}$(Fig. 1a, 4b and 6), which could be attributed to stronger hydrophobicity and interfacial preference of M-Gdm ${ }^{+}$ compared with $\mathrm{Gdm}^{+}$.

The distribution of $\mathrm{M}-\mathrm{Gdm}^{+} \theta_{\mathrm{CH}_{3}}$ angles in $3 \mathrm{~m}$ bromide and iodide solutions is also examined using the $2 \mathrm{D}$ orientationally resolved number density profiles $g\left(z, \theta_{\mathrm{CH}_{3}}\right)$ and $1 \mathrm{D}$ probability density profiles $\rho\left(\theta_{\mathrm{CH}_{3}}\right)$ as described previously. In general the orientational distributions of $\mathrm{M}-\mathrm{GdmBr}$ (Fig. 7 a, b) and M-GdmI (Fig. 7 c, d) are similar to those of M-GdmCl (Fig. 2 a, b). In the bulk, $\theta_{\mathrm{CH}_{3}}$ follows the uniform distribution $\rho\left(\theta_{\mathrm{CH}_{3}}\right)=1 / 2 \sin \theta_{\mathrm{CH}_{3}}$ (Fig. $\left.7 \mathrm{~b}, \mathrm{~d}\right)$. At the interface, distribution towards a certain tilt angle is observed both for bromide and iodide solutions. The peak of the interfacial $\theta_{\mathrm{CH}_{3}}$ distribution is $75^{\circ}$ for $\mathrm{M}-\mathrm{GdmBr}$ and $73^{\circ}$ for $\mathrm{M}-\mathrm{GdmI}$, both similar to the $72^{\circ}$ for $\mathrm{M}-\mathrm{GdmCl}$. A slight but perceptible change on the general shape of the probability 
a)

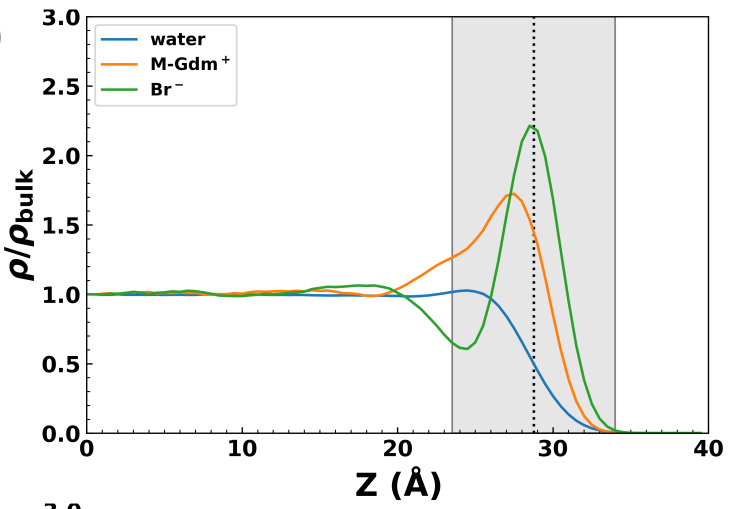

b)

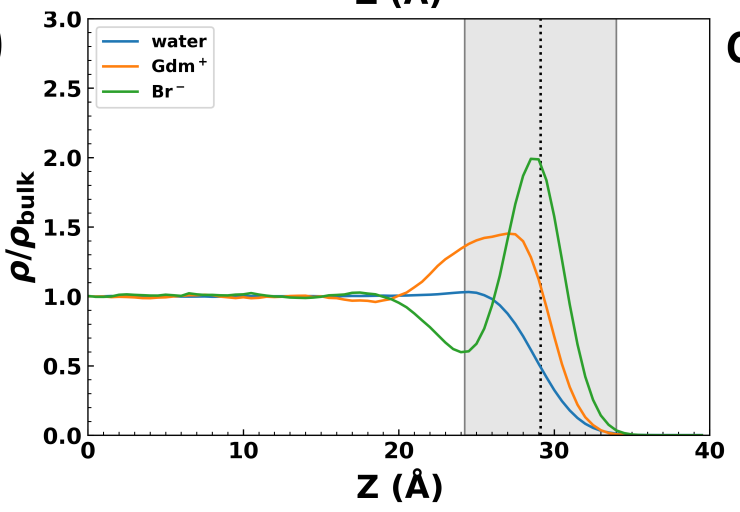

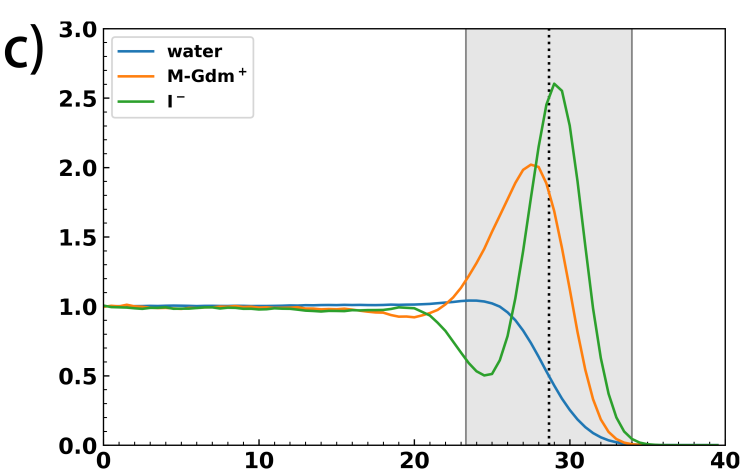

d)

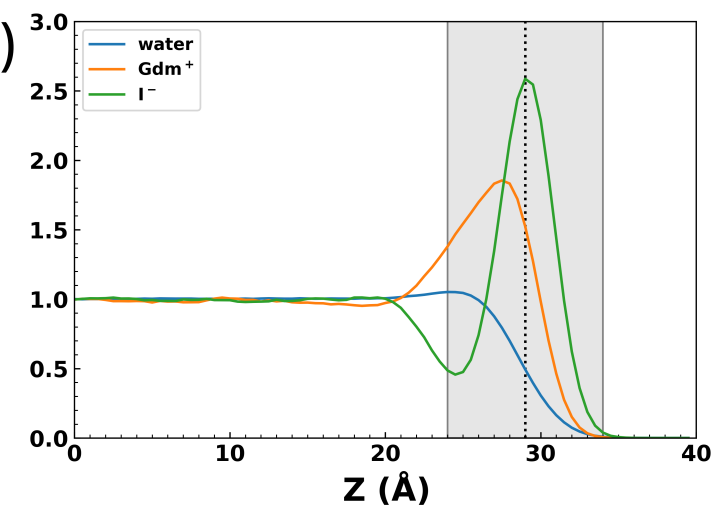

FIG. 6. Relative number density profiles of water, cation and anion from simulations of M-GdmBr (a), GdmBr (b), M-GdmI (c), GdmI (d) solutions at the molarity of $3 \mathrm{~m}$ using the Drude polarizable FF.

density function could be found with different counteranions. For example, the percentage of interfacial M-Gdm ${ }^{+}$ions whose methyl group point towards the air $\left(\theta_{\mathrm{CH}_{3}}<90^{\circ}\right)$ equals $60.3 \%$ for M-GdmBr and 57.6\% for M-GdmI, both smaller than the $65.6 \%$ for $\mathrm{M}-\mathrm{GdmCl}$. The percentage of interfacial $\mathrm{M}-\mathrm{Gdm}^{+}$ions with molecular plane tilted at an angle $<20^{\circ}$ relative to the surface plane decreases from $18.4 \%(\mathrm{M}-\mathrm{GdmCl})$ to $18.3 \%(\mathrm{M}-\mathrm{GdmBr})$ and $14.3 \%(\mathrm{M}-\mathrm{GdmI})$, as demonstrated in the $\theta_{\text {MP }}$ distributions (Fig. S4). Based on the distribution of $\theta_{\mathrm{CH}_{3}}$ for interfacial M-Gdm ${ }^{+}$, we could compute $D=\left\langle\cos ^{3} \theta_{\mathrm{CH}_{3}}\right\rangle /\left\langle\cos \theta_{\mathrm{CH}_{3}}\right\rangle$ values. The ensemble averaged $D$ equals $0.36 \pm 0.02$ and $0.29 \pm 0.02$ for $\mathrm{M}-\mathrm{GdmBr}$ and $\mathrm{M}-\mathrm{GdmI}$, both of which are smaller than the $0.45 \pm 0.01$ in the chloride solution. In summary, our simulatons predict that replacement of heavier halide anions will change the orientational distribution of $\mathrm{M}-\mathrm{Gdm}^{+}$at the air/water interface in such a way that can be readily measured by sum frequency generation experiments. 
$\mathrm{M}-\mathrm{GdmBr}$

a)

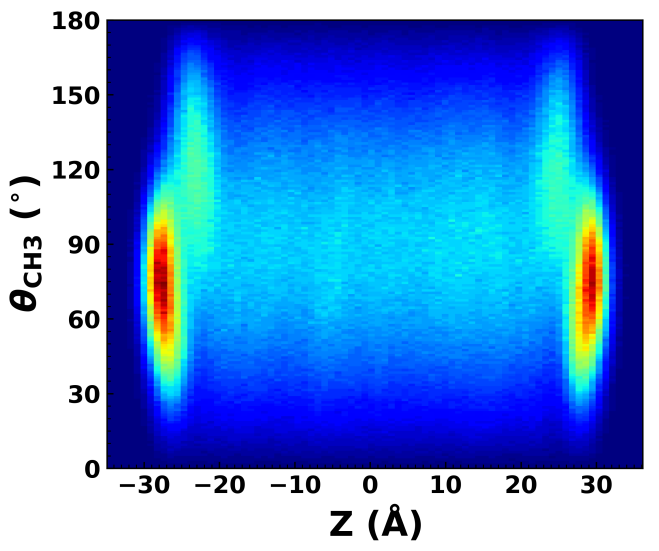

b)

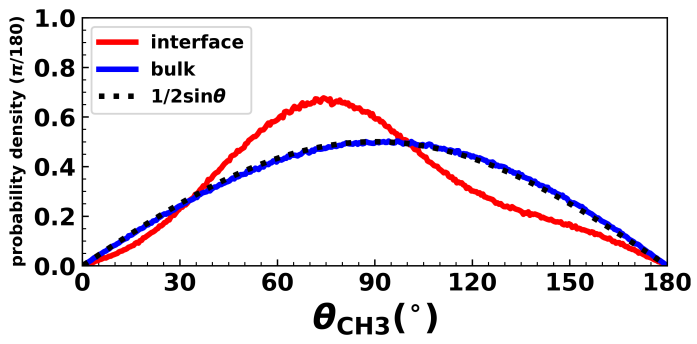

M-Gdml
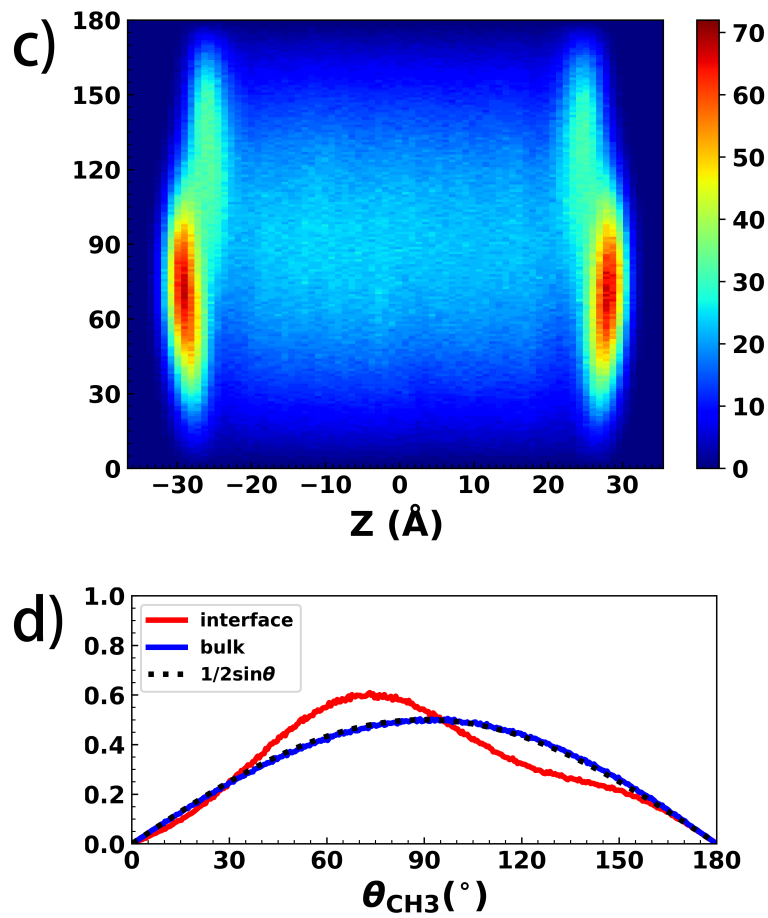

FIG. 7. The orientationally resolved number density profile $g\left(z, \theta_{\mathrm{CH}_{3}}\right)$ (top panels) of $\mathrm{M}-\mathrm{Gdm}^{+}$ions as a function of its orientation $\theta_{\mathrm{CH}_{3}}$ and its distance from box center $(z=0 \AA)$, together with the probabilty density profile of $\mathrm{M}-\mathrm{Gdm}^{+}$ions at the interface and in the bulk, as well as a standard function of $1 / 2 \sin \theta$ as a function of its orientation $\theta_{\mathrm{CH}_{3}}$ at the interface in the $\mathrm{M}-\mathrm{Gdm}^{+}$bromide ( $\mathrm{a}$ and $\mathrm{b}$ ) and $\mathrm{M}-\mathrm{Gdm}^{+}$iodide (c and d) solutions at the molarity of $3 \mathrm{~m}$ using the Drude polarizable FF.

\section{DISCUSSION AND CONCLUSIONS}

In this work we presented a MD simulation study of $\mathrm{M}-\mathrm{Gdm}^{+}$ions at the air/water interface employing a fully polarizable force field based on the classical Drude oscillator model. Our study is comparative in nature, as we also carried out MD simulations of the $\mathrm{Gdm}^{+}$ions, investigated effect of counteranions by simulating the chloride, bromine and iodide solutions, and compared with simulation results from the CHARMM additive force field. From these simulations, we quantified the interfacial preference of $\mathrm{M}-\mathrm{Gdm}^{+}$and dissected its orientational preference at the air/water interface. We characterized how polarization - especially the anisotropic polarization of molecular ions - impacts their interfacial properties. Our results also suggest that VSFG experiments constitute a sensitive approach to measure the orientation of molecular ions on the air/water 
interface.

One of the key motivations of this study is to compare simulation with experiment on the interfacial orientational preference of $\mathrm{M}-\mathrm{Gdm}^{+}$. The ensemble averaged $D$ value obtained from MD simulation with Drude FF $(0.45 \pm 0.01)$ agrees very well with the experimental measurement $(0.5 \pm 0.06)$. Two angles, $\theta_{\mathrm{CH}_{3}}$ and $\theta_{\mathrm{MP}}$, can be used to characterize the tilting of $\mathrm{M}-\mathrm{Gdm}^{+}$molecular plane on the surface, and our simulations show that they correlate well with each other. Using

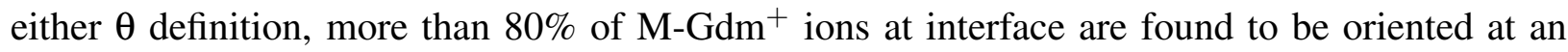
angle $>20^{\circ}$ relative to the surface plane, which is consistent with the conclusion drawn from the VSFG experimental study. ${ }^{24}$ We mainly use $\theta_{\mathrm{CH}_{3}}$ in this study because it directly connects with the VSFG measurement, while previous MD studies mostly use $\theta_{\text {MP }}$ or $\sin \theta_{\text {MP }}$ (for example Fig. 2 in Ref. 25 and Fig. S8 in Ref. 27). We note that it is the azimuthal angle that corresponds with a given ion orientation in a one-to-one way, and given a certain $\theta_{\mathrm{MP}}$ there can be many azimuthal

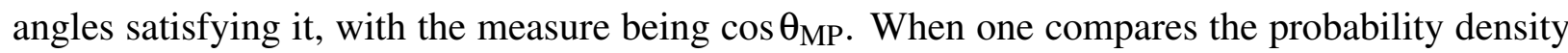
of different orientations, caution should taken regarding which angle is used and what the uniform distribution (null hypothesis) is.

We explored various polarization effects on the interfacial properties of ions in this study. In the Drude force field model, all non-hydrogen atoms are anisotropically polarizable. It is found that the polarizable heavy halide ions would accumulate on the air/water interface, while strong depletion is observed if they are modeled by an additive FF, consistent with many previous MD and theoretical studies. ${ }^{7,17,25}$ Analysis of the magnitude and direction of induced dipole moments demonstrates how ions move their charge distribution towards the liquid side of interface so as to keep charge hydrated and reduce the electrostatic self energy. Interestly, we reveal a direct coupling between the anisotropic molecular polarizabilty of $\mathrm{M}-\mathrm{Gdm}^{+}$ion and its orientation at the interface. For M-Gdm ${ }^{+}$, the in-molecular-plane component of polarizability is almost twice larger than the out-of-plane polarizability, such that charge redistribution is much easier to occur within the molecular plane. At the interface, ions tend to transfer their charges into the water phase. Simple ions such as $\mathrm{Cl}^{-}$point their induced dipole along the z-axis to maximize the charge redistribution. In contrast, molecular ions such as $\mathrm{M}-\mathrm{Gdm}^{+}$have to orient for the induction within molecular plane to be pointed towards the water phase. Although there are many factors that determine the overall orientational profile of interfacial $\mathrm{M}-\mathrm{Gdm}^{+}$, our study shows that polarization, 
in particular the anisotropic nature of molecular polarization, contributes favorably for M-Gdm ${ }^{+}$ to adopt a tilt angle at the air/water interface.

M-Gdm ${ }^{+}$and $\mathrm{Gdm}^{+}$are model compounds for Arginine side chain, so our study also serves as a validation on the corresponding Drude FF parameters in the highly inhomogeneous interface environment. Drude simulation results match all experimental data considered in this work. For the orientational preference of $\mathrm{M}-\mathrm{Gdm}^{+}$at the air/water interface, computational and experimental $D$ values agree within uncertainties. In terms of surface preference, the dependence of surface tension on solute concentration correlates well between simulation and experiment for $\mathrm{Gdm}^{+}$. It's interesting to note that $\mathrm{M}-\mathrm{Gdm}^{+}$and $\mathrm{Gdm}^{+}$, differing by one hydrophobic methyl group, exhibit qualitatively different surface preference in $3 \mathrm{~m}$ chloride solutions. The Drude polarizable FFs have been implemented in multiple MD engines including CHARMM, ${ }^{39}$ NAMD, ${ }^{61}$ Gromacs, ${ }^{62}$ LAMMPS ${ }^{63}$ and OpenMM. ${ }^{64}$ Our recent implementation of Drude FF in OpenMM allows simulations on GPUs and thus routine access to microsecond timescale for biomacromolecular systems. ${ }^{64}$ The Drude model is relatively efficient, with about 4 times slower than additive FFs (a factor of 2 from increased number of particles in simulation systems, and a factor of 2 from integration time step being halved to $1 \mathrm{fs}$ ), and is expected to provide more physical insights to study molecules in complicated electrostatic environments such as interfaces.

The orientation distribution of $\mathrm{M}-\mathrm{Gdm}^{+}$from MD simulations could be related with VSFG experiment through the $D$ value in Eq. (1). We note that $D$ value is quite sensitive to $\theta_{\mathrm{CH}_{3}}$ distribution. For example, the $1 \mathrm{D} \theta_{\mathrm{CH}_{3}}$ probability density profiles of $\mathrm{M}-\mathrm{Gdm}^{+}$at the interface in 3 m M-GdmCl, M-GdmBr and M-GdmI solutions are similar, but their corresponding $D$ values are clearly differentiable. Our calculations predict that the $D$ values for $\mathrm{M}-\mathrm{GdmBr}$ and $\mathrm{M}-\mathrm{GdmI}$ equal $0.36 \pm 0.02$ and $0.29 \pm 0.02$, respectively. In summary, our study supports that VSFG experiments are sensitive and effective methods to investigate the orientation distribution of molecular ions at the air/water interface. We expect combining experiments with simulations using high quality polarizable FFs will shed lights on the structure and dynamics of molecular ions at the interfaces. 


\section{ACKNOWLEDGEMENTS}

We thank Dr. Xiang Yu for valuable advice. The work is supported by National Natural Science Foundation of China (Grant No. 21803057) and by Zhejiang Provincial Natural Science Founda-

tion of China (Grant No. LR19B030001). We thank Westlake University Supercomputer Center for computational resource and related assistance. 
1 P. E. Mason, G. W. Neilson, C. E. Dempsey, A. C. Barnes, and J. M. Cruickshank, Proc. Natl. Acad. Sci. 100, 4557 (2003).

2 E. P. O’Brien, R. I. Dima, B. Brooks, and D. Thirumalai, J. Am. Chem. Soc. 129, 7346 (2007).

3 P. E. Mason, C. E. Dempsey, G. W. Neilson, S. R. Kline, and J. W. Brady, J. Am. Chem. Soc. 131, 16689 (2009).

4 C. Bissantz, B. Kuhn, and M. Stahl, J. Med. Chem. 53, 5061 (2010).

5 Y. N. Imai, Y. Inoue, and Y. Yamamoto, J. Med. Chem. 50, 1189 (2007).

6 J. Wang, J.-M. Choi, A. S. Holehouse, H. O. Lee, X. Zhang, M. Jahnel, S. Maharana, R. Lemaitre, A. Pozniakovsky, D. Drechsel, et al., Cell 174, 688 (2018).

7 P. Jungwirth and D. J. Tobias, Chem. Rev. 106, 1259 (2006).

8 D. J. Tobias and J. C. Hemminger, Science 319, 1197 (2008).

9 L. Onsager and N. N. Samaras, J. Chem. Phys. 2, 528 (1934).

10 L. Perera and M. L. Berkowitz, J. Chem. Phys. 95, 1954 (1991).

11 L. Perera and M. L. Berkowitz, J. Chem. Phys. 96, 8288 (1992).

12 P. B. Petersen and R. J. Saykally, Chem. Phys. Lett. 397, 51 (2004).

13 S. Ghosal, J. C. Hemminger, H. Bluhm, B. S. Mun, E. L. Hebenstreit, G. Ketteler, D. F. Ogletree, F. G. Requejo, and M. Salmeron, Science 307, 563 (2005).

14 H.-T. Bian, R.-R. Feng, Y. Guo, and H.-F. Wang, J. Chem. Phys. 130, 134709 (2009).

15 R.-R. Feng, H.-T. Bian, Y. Guo, and H.-F. Wang, J. Chem. Phys. 130, 134710 (2009).

16 S. Ou, Y. Hu, S. Patel, and H. Wan, J. Phys. Chem. B 117, 11732 (2013).

17 L. Sun, X. Li, Y. Tu, and H. Ågren, Phys. Chem. Chem. Phys. 17, 4311 (2015).

18 L. Sun, X. Li, T. Hede, Y. Tu, C. Leck, and H. Ågren, J. Phys. Chem. B 116, 3198 (2012).

19 C. Caleman, J. S. Hub, P. J. van Maaren, and D. van der Spoel, Proc. Natl. Acad. Sci. 108, 6838 (2011).

20 D. E. Otten, P. R. Shaffer, P. L. Geissler, and R. J. Saykally, Proc. Natl. Acad. Sci. 109, 701 (2012).

21 D. J. Tobias, A. C. Stern, M. D. Baer, Y. Levin, and C. J. Mundy, Ann. Rev. Phys. Chem. 64, 339 (2013).

22 R. Piazza, J. Cryst. Growth 196, 415 (1999).

23 E. Knipping, M. Lakin, K. Foster, P. Jungwirth, D. Tobias, R. Gerber, D. Dabdub, and B. FinlaysonPitts, Science 288, 301 (2000).

24 S. Strazdaite, J. Versluis, N. Ottosson, and H. J. Bakker, J. Phys. Chem. C 121, 23398 (2017). 
25 E. Wernersson, J. Heyda, M. Vazdar, M. Lund, P. E. Mason, and P. Jungwirth, J. Phys. Chem. B 115, $12521(2011)$.

26 T. Koishi, K. Yasuoka, S. Y. Willow, S. Fujikawa, and X. C. Zeng, J. Chem. Theo. Comp. 9, 2540 (2013).

27 S. Ou, D. Cui, and S. Patel, J. Phys. Chem. B 117, 11719 (2013).

28 S. Patel and C. L. Brooks III, J. Comp. Chem. 25, 1 (2004).

29 S. Patel, A. D. Mackerell Jr, and C. L. Brooks III, J. Comp. Chem. 25, 1504 (2004).

30 R. Breslow and T. Guo, Proc. Natl. Acad. Sci. 87, 167 (1990).

31 J. Werner, E. Wernersson, V. Ekholm, N. Ottosson, G. Ohrwall, J. Heyda, I. Persson, J. Soderstrom, P. Jungwirth, and O. Bjorneholm, J. Phys. Chem. B 118, 7119 (2014).

32 Y. Levin, Phys. Rev. Lett. 102, 147803 (2009).

33 Y. Levin, A. P. D. Santos, and A. Diehl, Phys. Rev. Lett. 103, 257802 (2009).

34 J. W. Ponder, C. Wu, P. Ren, V. S. Pande, J. D. Chodera, M. J. Schnieders, I. Haque, D. L. Mobley, D. S. Lambrecht, R. A. Distasio, M. Head-Gordon, G. N. Clark, M. E. Johnson, and T. Head-Gordon, J. Phys. Chem. B 114, 2549 (2010).

35 Y. Shi, Z. Xia, J. Zhang, R. Best, C. Wu, J. W. Ponder, and P. Ren, J. Chem. Theo. Comp. 9, 5430 (2013).

36 J. Huang, P. Lopes, B. Roux, and A. MacKerell, J. Phys. Chem. Lett. 5, 3144 (2014).

37 J. A. Lemkul, J. Huang, B. Roux, and A. D. MacKerell Jr, Chem. Rev. 116, 4983 (2016).

38 J. Huang, A. C. Simmonett, F. C. Pickard, A. D. MacKerell, and B. R. Brooks, J. Chem. Phys. 147, 161702 (2017).

39 G. Lamoureux and B. Roux, J. Chem. Phys. 119, 3025 (2003).

40 P. Lopes, J. Huang, J. Shim, Y. Luo, H. Li, B. Roux, and A. MacKerell, J. Chem. Theo. Comp. 9, 5430 (2013).

41 F.-Y. Lin, J. Huang, P. Pandey, C. Rupakheti, J. Li, B. Roux, and A. D. MacKerell, J. Chem. Theo. Comp. 16, 3221 (2020).

42 J. A. Lemkul and A. D. MacKerell Jr, J. Chem. Theo. Comp. 13, 2053 (2017).

43 J. A. Lemkul and A. D. MacKerell Jr, J. Chem. Theo. Comp. 13, 2072 (2017).

44 J. A. Lemkul and A. D. MacKerell Jr, J. Comp. Chem. 39, 2624 (2018).

45 D. S. Patel, X. He, and A. D. MacKerell Jr, J. Phys. Chem. B 119, 637 (2014). 
46 M. C. Small, A. H. Aytenfisu, F.-Y. Lin, X. He, and A. D. MacKerell, J. Comp.-Aid. Mol. Des. 31, 349 (2017).

47 H. Li, J. Chowdhary, L. Huang, X. He, A. D. MacKerell Jr, and B. Roux, J. Chem. Theo. Comp. 13, 4535 (2017).

48 J. Huang and A. MacKerell, Biophys. J. 107, 991 (2014).

49 Y. Ding, Y. Xu, C. Qian, J. Chen, J. Zhu, H. Huang, Y. Shi, and J. Huang, J. Comp.-Aid. Mol. Des. 34, $421(2020)$.

50 B. R. Brooks, C. L. Brooks III, A. D. Mackerell Jr, L. Nilsson, R. J. Petrella, B. Roux, Y. Won, G. Archontis, C. Bartels, S. Boresch, et al., J. Comp. Chem. 30, 1545 (2009).

51 F.-Y. Lin, P. E. Lopes, E. Harder, B. Roux, and A. D. MacKerell Jr, J. Chem. Inf. Model. 58, 993 (2018).

52 H. Yu, T. W. Whitfield, E. Harder, G. Lamoureux, I. Vorobyov, V. M. Anisimov, A. D. MacKerell Jr, and B. Roux, J. Chem. Theo. Comp. 6, 774 (2010).

53 G. Lamoureux, E. Harder, I. V. Vorobyov, B. Roux, and A. D. MacKerell Jr, Chem. Phys. Lett. 418, 245 (2006).

54 U. Essmann, L. Perera, M. L. Berkowitz, T. Darden, H. Lee, and L. G. Pedersen, J. Chem. Phys. 103, 8577 (1995).

55 W. G. Hoover, Phys. Rev. A 31, 1695 (1985).

56 G. J. Martyna, D. J. Tobias, and M. L. Klein, J. Chem. Phys. 101, 4177 (1994).

57 G. Lamoureux and B. Roux, J. Chem. Phys. 119, 3025 (2003).

58 W. L. Jorgensen, J. Chandrasekhar, J. D. Madura, R. W. Impey, and M. L. Klein, J. Chem. Phys. 79, 926 (1983).

59 J. G. Kirkwood and F. P. Buff, J. Chem. Phys. 17, 338 (1949).

60 A. Kumar, Fluid phase equilibria 180, 195 (2001).

61 W. Jiang, D. J. Hardy, J. C. Phillips, A. D. MacKerell Jr, K. Schulten, and B. Roux, J. Phys. Chem. Lett. 2, 87 (2010).

62 J. A. Lemkul, B. Roux, D. van der Spoel, and A. D. MacKerell Jr, J. Comp. Chem. 36, 1473 (2015).

63 A. Dequidt, J. Devemy, and A. A. Padua, J. Chem. Inf. Model. 56, 260 (2015).

64 J. Huang, J. A. Lemkul, P. K. Eastman, and A. D. MacKerell Jr., J. Comp. Chem. 39, 1682 (2018). 